\title{
Ileítis terminal por Yersinia enterocolitica: diagnóstico diferencial con la enfermedad de Crohn en un varón de 12 años
} Terminal ileitis due to Yersinia enterocolitica: differential diagnosis with Crohn's disease in a 12-year-old male

\author{
Dra. Stefanía Cian ${ }^{a}$ Dr. Omar Tabacco y Dr. Alejandro Costaguta
}

\section{RESUMEN}

Yersinia enterocolitica es un bacilo Gram-negativo causante de infección intestinal en los humanos. Se presenta con diferentes cuadros clínicos que obligan a descartar una variedad de etiologías, lo cual, a veces, hace difícil alcanzar un diagnóstico correcto en forma oportuna.

Se expone el caso de un varón adolescente con dolor en la fosa ilíaca derecha a partir de una ileítis terminal con hallazgos similares a la enfermedad de Crohn, que se diagnosticó, finalmente, como infección por Yersinia enterocolitica. Se destaca la utilidad de los diferentes métodos auxiliares empleados. Palabras clave: Yersinia enterocolitica, enfermedad de Crohn, ileitis terminal, niño.

\begin{abstract}
Yersinia enterocolitica is a gram-negative rod causing intestinal infection in humans. It shows different clinical pictures with many different etiologies to be ruled-out, which sometimes makes it difficult to reach a timely and correct diagnosis. We report the case of an adolescent boy presenting with right lower quadrant pain from terminal ileitis with endoscopic findings akin to Crohn's disease finally diagnosed as Yersinia enterocolitica, highlighting the usefulness of the different ancillary methods employed.

Key words: Yersinia enterocolitica, Crohn's disease, terminal
\end{abstract} ileitis, child.

http: / / dx.doi.org/10.5546/ aap.2020.e191

Cómo citar: Cian S, Tabacco O, Costaguta A. Ileítis terminal por Yersinia enterocolitica: diagnóstico diferencial con la enfermedad de Crohn en un varón de 12 años. Arch Argent Pediatr 2020;118(2):e191-e193. a. Servicio de Gastroenterología, Hepatología y Nutrición, Sanatorio de Niños, Rosario, Santa Fe, Argentina.

Correspondencia:

Dra.Stefanía Cian: stefaniacian@gmail.com

Financiamiento: Ninguno.

Conflicto de intereses: Ninguno que declarar.

Recibido: 15-4-2019

Aceptado: 11-9-2019

\section{INTRODUCCIÓN}

Yersinia enterocolitica es un bacilo Gramnegativo que pertenece a la familia Enterobacteriaceae. La infección, en los humanos, está asociada a diferentes formas de presentación de acuerdo con la edad del paciente. La manifestación más frecuente en los lactantes y los niños pequeños es la enteritis aguda, autolimitada y sin complicaciones. En los niños mayores y los adolescentes, predomina la afectación del íleon terminal, asociada, eventualmente, a adenitis mesentérica, que forma parte de los diagnósticos diferenciales del síndrome de fosa ilíaca derecha (FID), que incluye, entre otros, la enfermedad de Crohn y la apendicitis aguda. ${ }^{1-3}$

Se presenta el caso de un adolescente que consultó por dolor en la FID de varios días de evolución, en quien se diagnosticó, finalmente, ileítis por Yersinia enterocolitica. Se destaca la utilidad de los diferentes métodos complementarios para el diagnóstico diferencial.

\section{CASO CLÍNICO}

Un varón de 12 años concurrió a la consulta por un cuadro de dolor abdominal en la fosa ilíaca izquierda, asociado a disuria, de 20 días de evolución. El paciente no presentaba antecedentes de relevancia y vivía en una zona urbana, con adecuada disposición de excretas y agua corriente de red, sin contacto con animales.

Las pruebas bioquímicas, que incluían hemograma, plaquetas, función renal, glucemia y reactantes de fase aguda, así como una ecografía abdominal, mostraron resultados normales. El paciente recibió medicación sintomática y controles periódicos por consultorio externo. El dolor persistió de manera intermitente por varias semanas, tras lo cual se observó su migración a la FID, que aumentó de intensidad y agregó fiebre $\left(38{ }^{\circ} \mathrm{C}\right)$, por lo que se decidió la internación para completar los estudios. Una nueva ecografía abdominal mostró la presencia de adenomegalias en la FID, acompañadas, en el análisis de laboratorio, de un aumento en los 
reactantes de fase aguda (Tabla 1). La tomografía axial computada de abdomen confirmó los hallazgos ecográficos.

A las 48 horas del ingreso, debido a la persistencia del dolor y habiendo descartado una etiología quirúrgica, se realizó una endoscopía digestiva, que evidenció úlceras aftoides pequeñas rodeadas de mucosa normal en todo el trayecto del colon. La válvula ileocecal se franqueó sin dificultad y se recorrieron $\operatorname{los} 10 \mathrm{~cm}$ terminales del Íleon, en los que se observaron úlceras de bordes anfractuosos, cubiertas de fibrina, de 2 a $5 \mathrm{~cm}$ de longitud, que ocupaban toda la circunferencia del asa, con aumento de la nodularidad submucosa (Figura 1).

La endoscopía digestiva superior fue normal hasta la segunda porción duodenal. Debido a la sospecha diagnóstica de enfermedad inflamatoria intestinal (enfermedad de Crohn), se solicitó la realización del panel gastrointestinal FilmArray ${ }^{\circledR}$ y cultivos de material de biopsia y líquido intestinal para descartar una etiología infecciosa ante la posibilidad de requerir tratamiento inmunosupresor. El resultado del panel, recibido a los pocos minutos de haberse finalizado la endoscopía, fue positivo para Yersinia enterocolitica, confirmado 4 días después en el cultivo de la biopsia.

El paciente fue dado de alta al día siguiente de la endoscopía y controlado en forma ambulatoria hasta la resolución completa del cuadro, pocas

TaBla 1. Análisis de laboratorio de ingreso

\begin{tabular}{lc}
\hline Leucocitos & $14620 / \mathrm{mm}^{3}$ \\
Hemoglobina & $12,3 \mathrm{~g} / \mathrm{dl}$ \\
Hematocrito & $37,90 \%$ \\
Plaquetas & $179000 / \mathrm{mm}^{3}$ \\
Eritrosedimentación & $35 \mathrm{~mm}$ \\
Glucemia & $101 \mathrm{mg} / \mathrm{dl}$ \\
Uremia & $32 \mathrm{mg} / \mathrm{dl}$ \\
Bilirrubina total & $0,36 \mathrm{mg} / \mathrm{dl}$ \\
GGT & $13 \mathrm{U} / 1$ \\
GOT & $34 \mathrm{UI} / 1$ \\
GPT & $31 \mathrm{UI} / 1$ \\
Amilasemia & $48 \mathrm{U} / 1$ \\
PCR & $2,5 \mathrm{mg} / \mathrm{dl}$ \\
Calprotectina & Normal \\
Orina & Normal
\end{tabular}

Valores de laboratorio al momento de la internación. GGT: gamma glutamil transpeptidasa; GOT: glutamato oxalacetato transaminasa; GPT: glutamato piruvato transaminasa; PCR: proteína $C$ reactiva. semanas más tarde. El estudio histológico de las biopsias obtenidas en el íleon y el colon reveló un infiltrado inflamatorio mixto leve asociado a hiperplasia nodular linfoide reactiva no específica, con predominio en el îleon terminal.

\section{DISCUSIÓN}

La enteritis por Yersinia enterocolitica es una zoonosis de distribución mundial. El cerdo es el principal reservorio de cepas patógenas, que se eliminan a través de la materia fecal. Los humanos se infectan por el consumo de agua o alimentos contaminados con el microorganismo. La infección es más frecuente en los niños varones menores de 7 años y alcanza la máxima incidencia en la época invernal o en zonas de climas fríos. ${ }^{4}$

Después de la ingestión, los gérmenes son transportados al íleon terminal, donde se adhieren y penetran los enterocitos, para multiplicarse en las placas de Peyer y en los folículos linfáticos mesentéricos, lo que causa ulceración de la mucosa y adenomegalias regionales, como en el caso de nuestro paciente..$^{5}$ La infección, generalmente, es autolimitada, con curación en 1-3 semanas, sin mayores consecuencias. ${ }^{6,7}$

Debido a la amplia gama de manifestaciones clínicas con que puede presentarse la yersiniosis, el diagnóstico de esta enfermedad puede resultar

Figura 1. Aspecto endoscópico del ileon terminal: nodularidad submucosa aumentada, úlceras múltiples cubiertas por fibrina. Congestión y eritema de la mucosa, con áreas de menor afectación

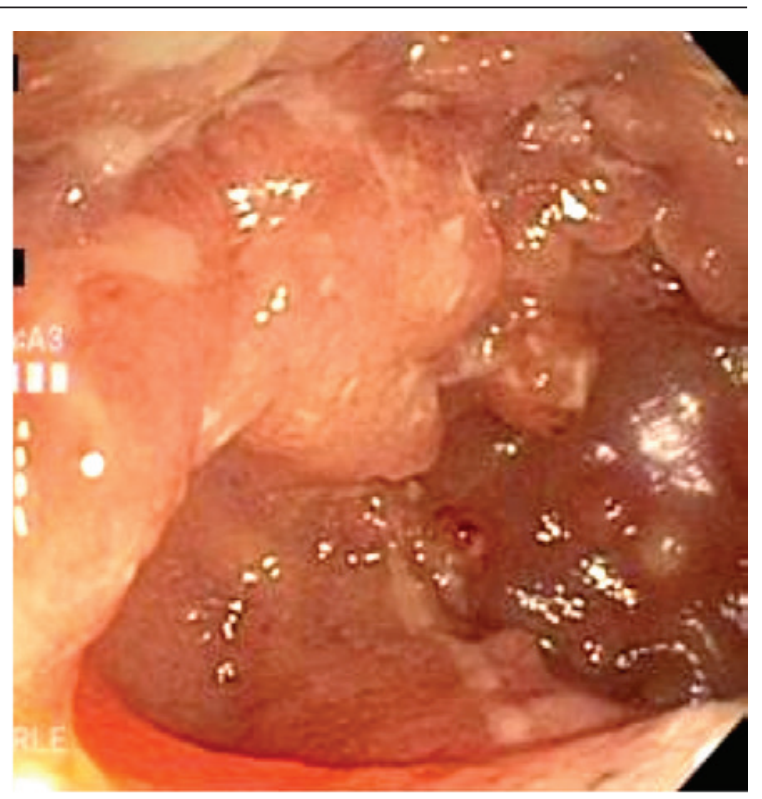


dificultoso y, a menudo, ser ignorado..$^{8,9} \mathrm{La}$ presentación como dolor abdominal localizado en la FID plantea diferentes etiologías posibles, desde cuadros de resolución quirúrgica, como una apendicitis, hasta patologías crónicas, como la enfermedad inflamatoria intestinal o de origen tumoral (linfoma), lo que resalta la importancia de un diagnóstico correcto. ${ }^{10-13}$

En el caso presentado, nuestro paciente refería un cuadro de dolor prolongado, que asoció fiebre el día previo a su internación. Debido a la ausencia de mejoría clínica, se decidió realizar el estudio endoscópico, cuyos hallazgos obligaron al diagnóstico diferencial con la enfermedad de Crohn.

La disponibilidad del panel FilmArray ${ }^{\circledR}$ permitió identificar, en pocos minutos, la presencia de Yersinia enterocolitica, lo que alentó la sospecha de una etiología infecciosa específica de la ileítis, confirmada días más tarde con el cultivo de la biopsia colónica. El panel FilmArray ${ }^{\circledR}$ es una prueba de laboratorio que utiliza técnicas basadas en un sistema de extracción de ácidos nucleicos y reacciones en cadena de la polimerasa (polymerase chain reaction; $P C R$, por sus siglas en inglés) múltiples. Detecta la mayoría de los microorganismos que pueden afectar el tracto digestivo, que incluyen virus, bacterias y parásitos, a partir de una muestra única de materia fecal. La rapidez con la que se reciben sus resultados (minutos) es una de las ventajas más destacadas, en comparación con el coprocultivo convencional. En nuestro paciente, esta característica fue particularmente útil, ya que orientó el tratamiento en forma rápida y segura. ${ }^{14}$

En conclusión, el síndrome de dolor en la FID comprende múltiples etiologías, con tratamientos muy diferentes. La ileítis por Yersinia enterocolitica debe ser considerada dentro del espectro de diagnósticos diferenciales. La correcta utilización de los métodos auxiliares puede contribuir a evitar el subdiagnóstico.

\section{REFERENCIAS}

1. Cohen M, Wayne Laney DJr. Diarrea infecciosa. En:Wyllie R, Hyams J. Gastroenterología pediátrica. $2 .{ }^{d a}$ ed. México, DF: McGraw Hill Interamericana; 2001.Págs.395-420.

2. Román Riechmann E, Barrio Torres J. Diarrea infecciosa. En: Argüelles Martín F, García Novo M, Pavón Relinchón P, Román Riechmann E, et al (ed.). Tratado de gastroenterología, hepatología y nutrición pediátrica aplicada de la SEGHNP. Madrid: Ergón; 2010.Págs.267-78.

3. González VázquezE, Quiñónez RamírezI. Falsa apendicitis: yersinia enterocolítica. Rev Digit Univ. 2005; 6(4):1-9.

4. Sojo Aguirre A, Álvarez PittiJ, Morteruel Arizkuren E, García González S, et al. Ileítis terminal por Yersinia enterocolitica en lactantes. An Pediatr (Barc). 2005; 63(6):555-7.

5. Arocena C, Galazka J, Picón T, Giachetto G. Apendicitis aguda, bacteriemia y artritis: manifestaciones de infección por Yersinia enterocolitica en un adolescente. Arch Pediatr Urug. 2012; 83(3):185-8.

6. Servicio Extremeño de Salud. Protocolo de vigilancia epidemiológica de la Yersiniosis. 2016. [Acceso: 12 de septiembre de 2019]. Disponible en: http:// www.areasaludbadajoz.com/SALUD_PUBLICA / EPIDEMIOLOG\%C3\%8DA/protocolo_yersiniosis_2016_ extremadura.pdf.

7. Pardo L, Mota M, Giachetto G, Parada M, et al. Adenitis mesentérica por Yersinia enterocolítica. Rev Med Urug. 2007; 23(4):383-6.

8. Molina Gutiérrez M, Martínez-Ojinaga Nodal E, Piñán Díez J, Rubio Aparicio P, et al. Acute Terminal Ileitis in Children. A retrospective Study in a Pediatric Emergency Department. Pediatr Emer Care. 2018; 25(4):249-51.

9. Winesett MP, Pietsch JB, Barnard JA. Yersinia enterocolitica in a child with intussusception. J Pediatr Gastroenterol Nutr. 1996; 23(1):77-80.

10. Jess T, Jess P. Acute terminalileitis, yersiniosis, and Crohn $\square \mathrm{s}$ disease: a long-term, follow-up study of the relationships. Eur J Intern Med. 2001; 12(2):98-100.

11. Fuchizaki U, Machi T, Kaneko S. Challenges and Images in GI. Gastroenterology. 2006; 131(5):1379.

12. Naddei R, Martinelli M, Strisciuglio C, D'Armiento M, etal. Yersinia enterocolitica ileitis Mimicking Pediatric Crohn $\square \mathrm{s}$ Disease. Inflamm Bowel Dis. 2017; 23(4):E15-6.

13. Macfarlane PI, Miller V. Yersinia enterocolitica mimicking Crohn $\square$ s disease. J Pediatr Gastroenterol Nutr. 1986;5(4):671.

14. Buss S, Leber A, Chapin K, Fey P, et al. Multicenter evaluation of the BioFire FilmArray gastrointestinal panel for etiologic diagnosis of infectious gastroenteritis. J Clin Microbiol. 2015; 53(3):915-25.s 